\title{
MEASUREMENT WITH BEAM OF THE DEFLECTING HIGHER ORDER MODES IN THE TTF SUPERCONDUCTING CAVITIES
}

\author{
Ch. Magne*, M. Jablonka, M. Lalot, V. Lethiers, M. Luong, O. Napoly, CEA/Saclay \\ N. Baboi, P. Castro, A. Gössel, M. Hüning, G. Kreps, M. Liepe, \\ M. Minty, S. Schreiber, D. Sertore, M. Wendt, DESY, 22603 Hamburg, Germany
}

\begin{abstract}
This paper reports on recent beam measurements of higher order modes in the TESLA Test Facility (TTF) accelerating modules. Using bunch trains of about $0.5 \mathrm{~ms}$ with $54 \mathrm{MHz}$ bunch repetition and up to $90 \%$ modulated intensity, transverse higher order modes are resonantly excited when the beam is offset and their frequency on resonance with the modulation frequency. With this method, the trapped modes can be excited and their counteraction on the beam observed on a wide-band BPM downstream of the module. Scanning the modulation frequency from 0 to $27 \mathrm{MHz}$ allows a systematic investigation of all possible dangerous modes in the modules.
\end{abstract}

\section{INTRODUCTION}

The transport of very low emittance beams along the TESLA linac [1] requires sufficient damping of the transverse higher order modes (HOM): to maintain a multibunch emittance smaller than the design single bunch emittance, damping factors $Q$ smaller than $10^{5}$ must be achieved in the TESLA superconducting cavities for the few modes, specially in the first two dipole passbands, with a large coupling impedance $R / Q$. Modes from higher passbands, above cutoff, are expected to couple efficiently to the HOM couplers in the beam tubes, except for a few so-called "trapped" modes concentrated in the central cells of the cavity. It is therefore important to verify with beam, that the HOM damping is adequate, and it is possible to detect unwanted trapped modes.

A first experiment performed at TTF in 1998 [2] showed the existence of a highly coupled mode of the third dipole passband, at about $2585 \mathrm{MHz}$ frequency, with a $Q$-factor in excess of $10^{5}$ in several cavities. We report on the second experiment which took place last spring with the aim of investigating thoroughly the first 6 dipole mode passbands of the TESLA 9-cell superconducting cavities (see Table 1). The principle of the experiment [3] is to excite HOMs resonantly with the side-band frequencies generated on both sides of the bunch harmonics by modulating the bunch charge of a long train of bunches. Once excited, the HOM deflects the bunch train and a signal can be observed with a BPM downstream of the module. By scanning the modulation frequency $f_{m}$ from 0 to half the bunch frequency $f_{b}$, all HOMs will resonate with the bunch train when their frequency $f_{\mathrm{HOM}}$ coincides with one of the side-band frequencies $n f_{b} \pm f_{m}$, with $n$ an integer. To better separate the HOMs resonances in the modulation frequency space, it is therefore important to use the highest possible bunch frequency $f_{b}$.

\section{EXPERIMENTAL SET-UP}

The layout of the experimental set-up is shown in Fig. 1. The first TTF accelerating module includes eight 9-cell $1.3 \mathrm{GHz}$ superconducting cavities. During the experiment the RF gun delivered up to $5 \mathrm{~mA}$ of beam current at a pulse duration of $500 \mu$ s with bunch frequency of $54 \mathrm{MHz}$ with $10-90 \%$ charge modulation. The specific experimental setup includes a 'dog-leg' magnet (half a chicane) in front of the accelerating module providing a steady horizontal offset in the range from 0 to $20 \mathrm{~mm}$ and, a broadband cavity BPM located about $6 \mathrm{~m}$ downstream of the accelerating module exit, to measure individual positions of the bunches. Entering the module, beam energy was about 16 $\mathrm{MeV}$. The gradients of the eight cavities in the module were set to a minimum value of $2 \mathrm{MV} / \mathrm{m}$ in order to maximise the sensitivity of the beam to HOM deflections.

\subsection{Modulation of the laser beam}

The TTF injector is based on a laser driven rf gun.[5] Short UV laser pulses illuminate a CsKTe photocathode and produce a train of electron bunches with high peak current. The laser system is described in detail elsewhere.[6]

The electron beam charge is modulated along the pulse train by an appropriate modulation of the laser pulse train itself. A Pockels cell is used in combination with a polarizer to modulate the laser pulse train in time. The function of the Pockels cell is to rotate the linear polarization of the laser beam. The amount of rotation depends on the

Table 1: Passband limits of the lowest dipole modes calculated[4] for the design shape of TESLA 9-cell cavities.

\begin{tabular}{|c|c|c|}
\hline Band \# & $f_{0} / \mathrm{GHz}$ & $f_{\pi}$ \\
\hline 1 & 1.6197 & 1.7920 \\
2 & 1.8877 & 1.8261 \\
3 & 2.5782 & 2.4713 \\
4 & 3.0333 & 2.8134 \\
5 & 3.1231 & 3.0802 \\
6 & 3.3419 & 3.3595 \\
\hline
\end{tabular}

*christian.magne@cea.fr 


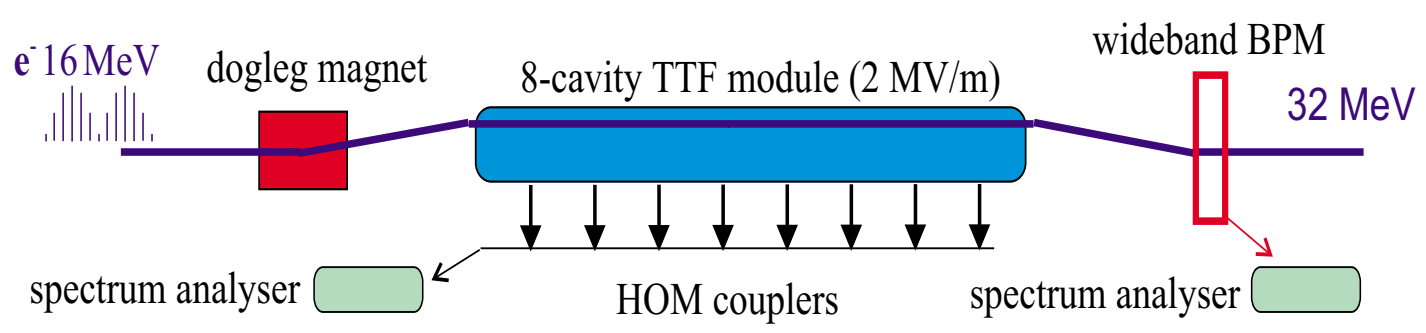

Figure 1: Experimental set-up.

high voltage applied to the Pockels cell crystal. The polarizer following acts as an analyzer: its transmission depends on the angle of the laser beam polarization and its principal axes. An external rf signal adjustable in the frequency range between $100 \mathrm{kHz}$ and $27 \mathrm{MHz}$ is input to the high voltage driver of the Pockels cell. Thus, the high voltage over the cell and in consequence the polarization of the laser beam is modulated according to the frequency and amplitude of this rf signal. The modulated pulse train is then amplified by a linear chain of four Nd:YLF amplifiers. Although the conversion into UV by a LBO and a $\mathrm{BBO}$ crystal is nonlinear, the modulation of the UV beam is mainly sinusoidal (about $90 \%$ ).

\subsection{Measurement of the BPM signal}

The deflection caused by the HOMs is measured with the difference signal from the wideband BPM. Due to the pulsed structure of the beam and the large bandwidth of the BPM the beam induces a signal at all harmonics of the bunch repetition rate and at the modulation sidebands.

The deflection caused by the HOMs can be separated from other signals using a variable filter with the center frequency set to the sideband frequency. For this purpose a spectrum analyser in zero span mode was used. A filter bandwidth of $1 \mathrm{MHz}$ delivered good separation and had an intrinsic time constant that is reasonably smaller than the observed time constants of the modes. With the chosen parameters the noise floor was found at $-60 \mathrm{dBm}$. Except for the position modulation due to the HOM the difference signal also contains the amplitude modulation used for the excitation of the modes. For the weaker modes the position modulation was smaller than the amplitude modulation depending on the position offset. To increase the sensitivity the modulation is stopped after the first $300 \mu s$ of the $500 \mu \mathrm{s}$ beam pulse. For the last $200 \mu \mathrm{s}$ the beam current is left unmodulated: then a signal with the appropriate frequency can only be caused by the deflecting HOM. An exponentially decaying signal is expected with a time constant given by the loaded $Q$ of the HOM. This can be seen in Fig. 2 for the modulation frequency $f_{m}=23.773 \mathrm{MHz}$.

For the measurement of $R / Q$ it is necessary to measure the exciting current. For this purpose the BPM sum signal was used in the same way. The calibration was done using the unmodulated beam measuring the harmonic of the

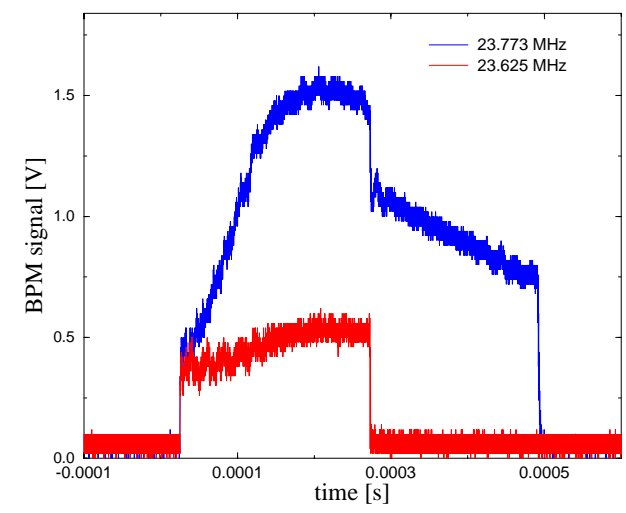

Figure 2: Resonant (23.773 MHz) and non-resonant (23.625 MHz) BPM signals.

bunch frequency. For the experiment the current was measured by the appropriate sideband taking into account the factor of 2 between bunch harmonic and sideband.

\subsection{Measurement of the HOM-coupler signals}

Picking-up the HOM signal at the HOM-coupler allows to identify the resonating cavity and to measure the excited HOM frequency and its time response. Every 9-cell cavity is equipped with one HOM-coupler at each end, with a relative angle of $105^{\circ}$, yielding a total of $16 \mathrm{HOM}$-coupler signals. A set of microwave coaxial switches (RLC-Transco SP6T) was used to connect them to the HP E8563E spectrum analyzer. To reduce reflections in the not-connected branches, all HOM-signals where passed through $10 \mathrm{~dB}$ attenuators. A VXI-based switch control unit was established for remote control.

With the fixed bunch frequency $f_{b}$ and the known charge modulation frequency $f_{m}$ the search for HOM excited frequencies is limited to a finite number of frequencies $f_{\mathrm{HOM}}=n f_{b} \pm f_{m}$ (with $n$ being an integer). Therefore the spectrum analyzer was set to zero span, realizing a time-domain decay measurement. Resolution and video bandwidth where both set to a moderate value $(100 \mathrm{kHz})$, in order to estimate the $Q$-value from the decay time of the excited mode. A semi-automatic measurement routine (VEEPro) controls the instruments and sets the center frequency of the spectrum analyzer to $n f_{b} \pm f_{m}$. Examples of signals taken are shown in Figs.(3-5) for HOM identified 


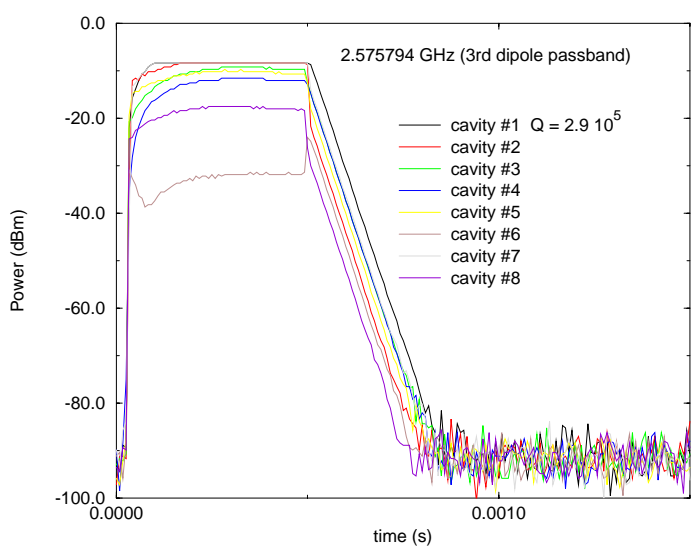

Figure 3: HOM coupler signal at $2.5757 \mathrm{GHz}$ for a $3^{\text {rd }}$ dipole passband mode.

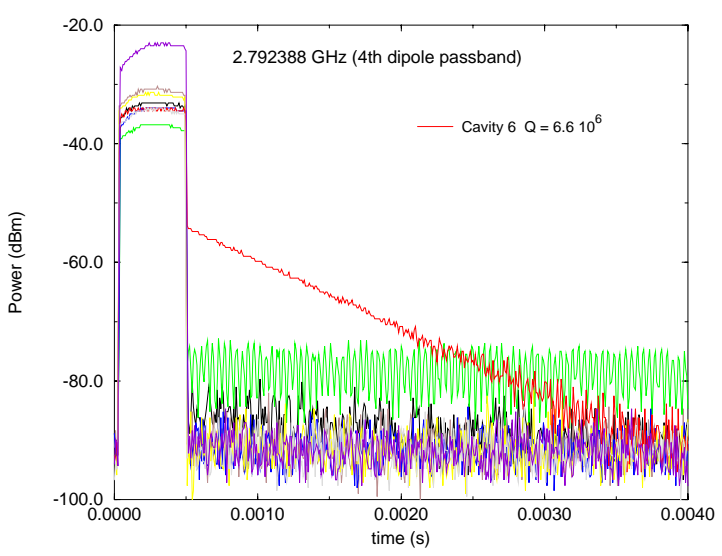

Figure 4: HOM coupler signal at $2.7923 \mathrm{GHz}$ for a $4^{\text {th }}$ dipole passband mode.

respectively in the $3^{\text {rd }}, 4^{\text {th }}$ and $5^{\text {th }}$ diple passbands.

\section{DISCUSSION}

While the analysis of the results is still underway, some conclusions can already be drawn from this experiment.

- Three different patterns of HOM signals are observed: in Fig. 3 the mode spans the entire 8-cavity module, in Fig. 4 the mode is isolated in a single cavity, and in Fig. 5 the same frequency is excited with the same damping time in two distant cavities and with a different damping time in a third cavity. The first two can be explained by propagating versus non-propagating modes, but the last pattern lacks an interpretation.

- Most modulation frequencies $f_{m}$ co-excite several HOMs belonging to different passbands, very often including a quadrupolar mode with a powerful HOM signal. This is certainly related to the finite width of the sideband and to the large beam offset $(19 \mathrm{~mm})$ in the module. To avoid this problem, the linear dependence of the signals with the beam offset should be checked systematically.

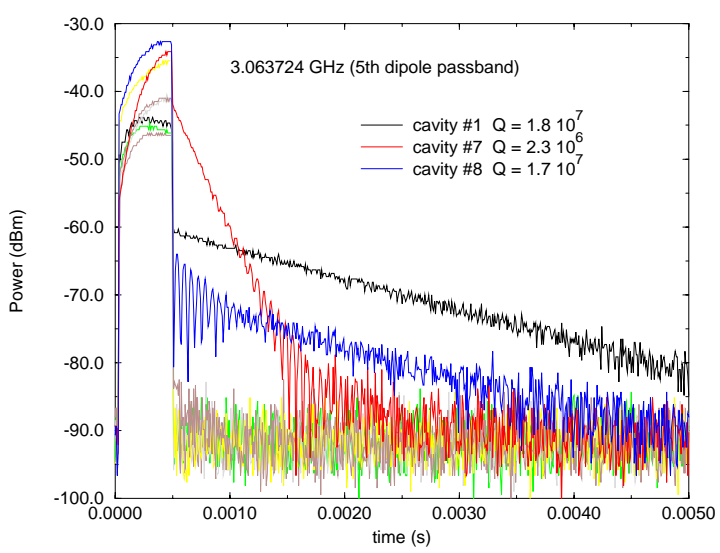

Figure 5: HOM coupler signal at $3.0637 \mathrm{GHz}$ for a $5^{\text {th }}$ dipole passband mode.

- While the $Q$-factor can be measured from the time decay of the HOM signals, the impedance $R / Q$ of dipole modes must be infered from the measurement of the beam displacements at the BPM. Unfortunately, when several co-excited modes contribute to this displacement, the BPM signal cannot be used. Moreover, both horizontal and vertical beam offsets are needed to drive the HOM excitation in order to access the trigonometric factor due to the mode polarization.

\section{ACKNOWLEDGEMENT}

We thank I. Will and A. Liero (Max-Born Institut, Berlin) for their effort to make the modulation of the laser possible, P. Michelato (LASA, Milano) for the preparation of the CsKTe cathode, and the TTF shift crew for their tremendous engagement for this experiment.

\section{REFERENCES}

[1] R. Brinkmann, K. Flöttmann, J. Roßbach, P. Schmüser, N. Walker and H. Weise (eds.), "TESLA Technical Design Report, Part II The Accelerator", DESY 2001-011, http://tesla.desy.de/new_pages/TDR_CD/PartII/accel.html

[2] S. Fartoukh et al., Proc. of the 1999 Particle Accelerator Conference, New York, USA, p.922.

[3] S. Fartoukh, "A New Method to Detect the High Impedance Dipole Modes of TESLA Cavities", Saclay Preprint, DAPNIA/SEA-98-18.

[4] R. Wanzenberg, "Monopole, Dipole and Quadrupole Passbands of the TESLA 9-cell Cavity", work in progress

[5] S. Schreiber, Proc. of the Europ. Particle Acc. Conf. EPAC2000, Vienna, Austria, p. 309.

[6] S. Schreiber, D. Sertore, I. Will, A. Liero, W. Sandner, "Running Experience with the Laser System for the RF Gun based Injector at the Tesla Test Facility Linac", Nucl. Instrum. Meth. A445 (2000) 427-431. 